\title{
Clusters of Defects as a Possible Origin of Random Telegraph Signal in Imager Devices: a DFT based Study
}

\author{
A. Jay, A. Hémeryck, F. Cristiano \\ LAAS CNRS, \\ Université de Toulouse, Toulouse, France
}

\author{
N. Richard \\ CEA, DAM, DIF, \\ Bruyères-le-Châtel, F-91297 Arpajon, France
}

\author{
D. Rideau, P.L. Julliard \\ ST Microelectronics, \\ Crolles, France
}

\author{
V. Goiffon, A. LeRoch \\ ISAE-SUPAERO,
}

Université de Toulouse, F-31055 Toulouse, France

\author{
L. Martin Samos, S. De Gironcoli \\ CNR-IOM, Democritos National Simulation Center, \\ Istituto Officina dei Materiali, c/o SISSA, IT-34136 Trieste, Italy
}

\begin{abstract}
The origin of the random telegraph signal (RTS) observed in semiconductors-based electronic devices is still subject to debates. In this work, by means of atomistic simulations, typical clusters of defects as could be obtained after irradiation or implantation are studied as a possible cause for RTS. It is shown that:

(i) a cluster of defects is highly metastable,

(ii) it introduces several electronic states in the band gap,

(iii) it has an electronic cross section much higher than the one of point defects.

These three points can simultaneously explain why an electronhole generation rate can switch with time, while respecting the experimental measurement.
\end{abstract}

Index Terms-Dark current, random telegraph signal, defects, electronic states, cross sections.

\section{INTRODUCTION}

Nowadays, most of the imagers are composed by a large volume of semiconductor that collects photo-generated carriers. It is surrounded by $\mathrm{SiO}_{2}$ oxide and both materials exhibit defects in their structure. This paper is focused on bulk crystalline defects that are known to play a dominant role in imagers' dark current. In fact, their presence in the crystal induces additional electronic states that can be located in the band gap, giving rise to the well known non-radiative multiphonon Shockley-ReadHall generation rate (SRH [1]-[3]). This parasitic electron-hole generation can be detrimental to the performances of most of microelectronic Silicon-based devices. In image sensors, this noise is called "dark current" (DC) since it occurs even in the dark, when no light excites the electrons. As known, this thermally activated current can be either constant or can switch between two or more values [4]. This later is referred as random telegraph signal (DC-RTS), because the switches between several identical DC values occur in an unpredictable way. A RTS is characterized by its difference of amplitudes between two values $\triangle D C=D C_{1}-D C_{2}$ (elec./s) and by the ratio of lifetime spent in each DC value $r_{T}=T_{1} / T_{2}$.

978-1-6654-0685-7/21/\$31.00@2021 European Union
While it is clearly stated that DC-RTS are due to the presence of defects, there is no consensus on the basic mechanisms that produce them. Several hypotheses have been proposed in the literature, such as the change of the generation center charge state [5]-[7], the change of a modulator charge state near a generation center [8], [9], or the change of the atomic structure of simple defects such as the P-V [10]-[13] and the O-V [14], [15] centers. However, it has been shown [16] that none of these hypotheses can explain simultaneously the experimental observations listed above:

-Observation a [17]: The characteristic lifetime of RTS amplitudes spans from microseconds to months, even years, and depends on the duration of the observation and of the time step during which the electrons are collected.

-Observation $b$ [4]: RTSs can have more than two amplitudes. Up to ten have been measured with the certainty that they do not originate from separate defects.

-Observation c [18]: For simple dark currents that do not oscillate, all activation energies above the mid-gap are observed. However, the activation energies of RTS are mainly at midgap. Some of them are slightly higher than mid-gap but this number is negligible.

-Observation $d$ [19]: The variations of the RTS amplitudes between two levels are more than thousands of electrons per second. This is greater by two orders of magnitude than the generation rate calculated for the worth known point defect (the PV center) with the SRH theory [20]:

$$
D C \sim \frac{\sigma v_{t h} n}{2 \cosh \left(\frac{E_{t}-E_{m g}}{k_{B} T}\right)}
$$

where $v_{t h}$ is the electron thermal velocity, $n$ is the carrier concentration, $E_{t}$ and $E_{m g}$ are respectively the trap and midgap energy levels. For the PV center, $E_{t}$ is $0.09 \mathrm{eV}$ above the mid-gap and $\sigma=7 \times 10^{-15} \mathrm{~cm}^{2}$ [21], giving about 20 electrons per second at $300 \mathrm{~K}$.

-Observation e [22]: The presence of RTS is not affected by the type of dopants, nor by their concentration. This permits 
to reduce the search for potential candidates at the origin of RTS to defects composed of pure silicon.

-Observation $f$ [23]: The RTS time constants and their amplitude differences are not affected by the variation of the electric field as long as the field is small enough to avoid the tunneling effect [24]. This eliminates from the remaining candidates the previous assumptions as well as the defects for which the electric field changes the electronic levels, such as the rotation of a defect parallel or orthogonal to the field.

-Observation $g$ [25]: After a long time, most of the $r_{T}$ ratios between two time constants are lower than 100. This implies that the energy barriers and the inverse energy barriers required to switch from one RTS level to the other are quite symmetric with a difference of less than $0.1 \mathrm{eV}$ at $300 \mathrm{~K}$.

Another hypothesis [16] is that some defects may change their atomic structure with time, resulting in a change in their electronic level in the band gap, and then in their electrons and holes generation rate. Although this hypothesis cannot be disproved by any of the above observations, no defect has been found that resolves them all simultaneously. We follow this latter hypothesis and discuss the possibility that bulk defect clusters are the perfect candidate for the origin of the RTS observed in typical image sensors. We study with first-principles calculations the metastability and electronic activity of a typical cluster composed by many interstitial atoms, as could be obtained after implantation processes. The same trends are expected in amorphous pockets, as could be obtained after irradiation, or in any other cluster that is sufficiently large and stable.

\section{Methodology}

The generation of a realistic agglomeration of interstitial silicon atoms is the key to simulate a relevant dark current. To the best of our knowledge, extended clusters of defects have only been studied with empirical methods, but never in a rigorous atomistic simulation. In this work, the multiscale strategy shown in [26], [27] is improved and completed to finally determine the electron-hole generation rate of a given cluster.

First, 14 interstitial atoms (14-I) have been randomly placed in a box of 512 crystalline silicon atoms governed by a Tersoff interatomic potential. 14 appears to be one of the most relevant intermediate number between the 4-I structure [28] (and its multiples 8-I, 12-I [29]) that is too stable to change its atomic structure at ambient temperature, and the apparition of the rodlike structures [30] that required a huge number of interstitial atoms. Other cluster sizes could also be used without changing the conclusions. Second, a molecular dynamics simulation at $900 \mathrm{~K}$ is performed during 1 ps to make them diffuse and agglomerate with LAMMPS [31]. Third, the kinetic activation relaxation technique [32] is used to simulate their aging and to ensure a stable configuration. Within this step, two 4I structures appear in the final cluster. Fourth, the system is relaxed and metastable atomic configurations are obtained using the ARTn-DFT coupling [33], allowing to access for the first time to the details of the geometry of this large defect at the $a b$ initio level. Fifth, the thermally activated electron-hole generation rate produced by the defect is calculated within the multiphonon approach [1]. According to this approach, the transition from the initial valence/conduction bands to the final localized defect state becomes possible when the $H$ Hamiltonian of the system is sufficiently modified by a phonon-induced change in atomic positions. This transition rate $\Gamma_{i \rightarrow f}$ has been calculated using the Fermi golden rule within the static coupling [34]: $\quad \Gamma_{i \rightarrow f} \sim$

$$
|\underbrace{\left\langle\phi_{i}\left|\frac{\partial H}{\partial \mathbf{Q}}\right| \phi_{f}\right\rangle}_{\text {elec overlap }} \underbrace{\left\langle\chi_{i m}|\mathbf{\Delta} \mathbf{Q}| \chi_{f n}\right\rangle}_{\text {ionic overlap }}|^{2}\left(E_{i}^{e l}-E_{f}^{e l}+E_{i}^{i}-E_{f}^{i}\right)
$$

where $Q$ is the direction of the atomic displacement induced by the electronic excitation, $\phi(\chi)$ and $E^{e l}\left(E^{i}\right)$ are respectively the electronic (ionic) eigenvectors and eigenvalues of electronic Hamiltonian $H^{e l}$ ( $H^{i o n}$ ) corresponding to the initial $(i)$ and final $(f)$ states. In practice, $H^{e l}$ and $H^{i o n}$ form an uncoupled system after the static and adiabatic approximations on the full Hamiltonian. $E^{e l}$ is calculated as the DFT energy of the $H^{e l}$, whereas $E^{i o n}=n \hbar \omega$, where $\omega$ is the phonon frequency and $n(m)$ the excitation state of the quantum harmonic oscillator. This transition rate is the key value to determine the electronic cross sections used in the SRH theory [2], [3].

\section{RESULTS}

Metastability: From the ground state configuration of the 14-I cluster, we have identified a large number of metastable states. Thus, the global atomic configuration changes in time, respecting the Boltzmann weight of each configuration and the corresponding kinetic barriers [35]. The energy barriers that have been found range from $0.6 \mathrm{eV}$ to over $2 \mathrm{eV}$ (Fig. 1). This wide range implies that for any temperature or time of

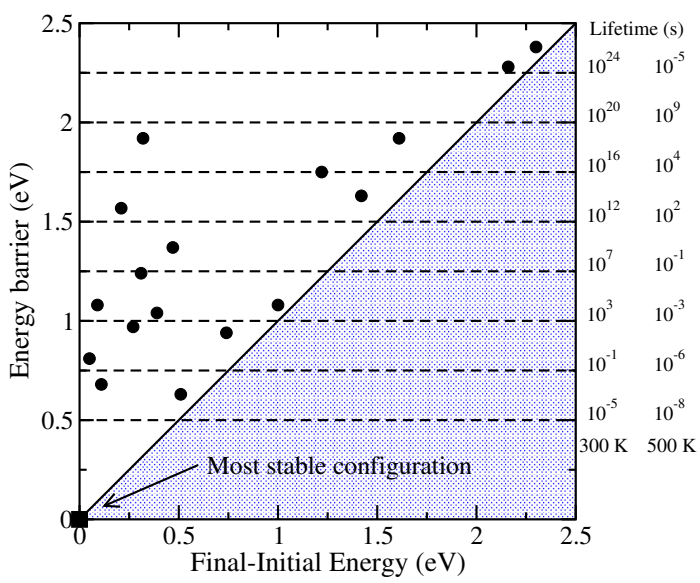

Fig. 1: Energy landscape of cluster 14-I and corresponding switch transition times (Lifetime) as a function of the energy barrier at $300 \mathrm{~K}$ and $500 \mathrm{~K}$. Each point represents a metastable structure. The most stable configuration is taken as a reference (black square at the origin). 
measurement, a change of atomic configuration is possible. Each of them has its own energy levels in the band gap and generates its own dark current amplitude, different from the others, leading to a possible DC-RTS signal. The large number of metastable states and energy barriers is consistent with the observations $a$ and $b$ listed in the introduction.

Electronic states: Cluster 14-I introduces a large number of electronic states into the band gap (Fig. 2). When many states are present in the band gap, the SRH generation rate is dominated by the transition for which the electronic trap energy is the closest to the mid gap, as it is the one for which the term $E_{t}-E_{m g}$ in the exponential of Eq. 1 is the smallest. The presence of so many traps in the band gap increases the probability that one of them is located as close as possible to the mid-gap and thus reduce $\left|E_{t}-E_{m g}\right|$. From the multiple simulations performed, we observed that the larger the cluster, the more electronic states it contains. Thus, large defect clusters have electronic activation energies that are almost mid-gap. Combined with their high metastability, and in contrast with point defects which are more stable and with a lower probability to have midgap activation energies, this is consistent with observation $c$.

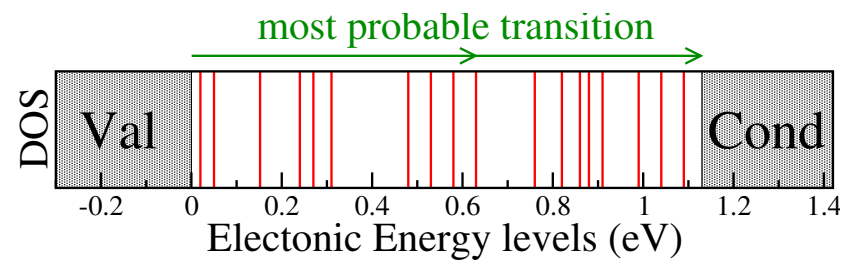

Fig. 2: Si band gap and the localised defect states (red) induced by the cluster 14-I (using DFT-HSE). Arrows indicate the closest to mid-gap transition.

Cross sections: The difference between the $\Gamma_{i \rightarrow f}$ generation rate of a point defect and that of a defect cluster is due to the small and extended $\Delta \mathrm{Q}$ displacement induced by electronic excitation (Fig. 3a) and the large spatial extension of the localized electronic wavefunctions of the defects (Fig. 3b).

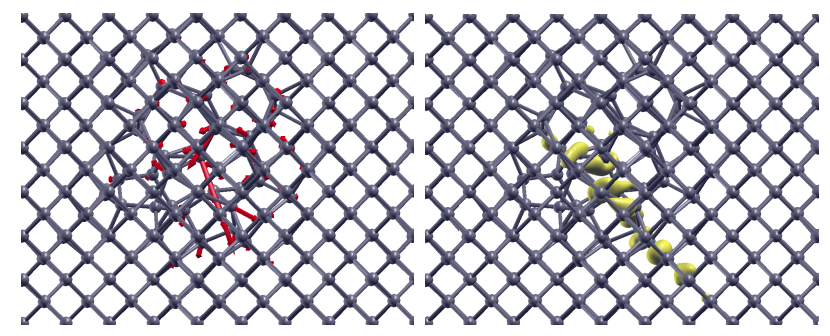

(a) Displacement $\Delta \mathrm{Q}$

(b) Elec. wavefunction $\phi_{i}$

Fig. 3: The 512 atoms of crystalline $\mathrm{Si}+$ cluster 14-I supercell. (a) Red arrows indicate the total displacement $\Delta Q$ vector $(\times 40)$. (b) Yellow surface is the $50 \%$ isovalue of the highest occupied electronic wave function.
For example, in a divacancy (not shown), the variation of atomic positions induced by the addition of a charge is about $0.3 \AA$ and is distributed over only two atoms. It decreases to $0.01 \AA$ in our 14 -I cluster and is distributed over all atoms in the cluster. Similarly, the electronic wave functions of divacancy are localized around two atoms, whereas they are extended in the case of a cluster.

These have three main impacts on Eq. 2. First, due to the small $\Delta \mathrm{Q}$, only a small number of phonons are needed to obtain such an amplitude (i.e the Huang-Rhys factor is small and lower than one), which means that the effective cross section does not depend on the temperature. Second, increasing the spatial extension of the localized electronic wave functions increases their overlap with the delocalized bulk states of the silicon material $\left\langle\phi_{i}\left|\frac{\partial H}{\partial \mathbf{Q}}\right| \phi_{f}\right\rangle$. Third, the very small atomic displacement required for the transition increases the slope of this derivative. With regards to the ionic overlap $\left\langle\chi_{i m}|\Delta \mathbf{Q}| \chi_{f n}\right\rangle$, it has only a minor impact on Eq. 2.

In comparison to the divacancy, we estimated that the highest electronic overlap shown in Fig. 4 increases by about two orders of magnitude. Combined with the square in the Fermi's golden rule, this implies that the cross section is about four orders of magnitude higher for clusters than for point defects, which is consistent with observation $d$.

Electronic overlaps calculation: The stable atomic positions and their corresponding energy have been calculated in their neutral state and in the excited state with one electron removed. All intermediate energies between these two configurations were then calculated in each charge state to form the two red parabolas of Fig. 4. For each $\mathbf{Q}$ position, the dot product between the perturbed electronic wave function of the last occupied defect state $\phi_{f}(\mathbf{Q})$ and each initial valence state $\phi_{i}(0)$ was then calculated. Each slope represents the contribution of the corresponding valence state in the electronic part of Eq. 2 using the relation: $\left\langle\phi_{i}\left|\frac{\partial H}{\partial \mathbf{Q}}\right| \phi_{f}\right\rangle=$ $\left(\epsilon_{f}-\epsilon_{i}\right) \frac{\partial\left\langle\phi_{i} \mid \phi_{f}\right\rangle}{\partial \mathbf{Q}}$. The dot product is zero at $\mathbf{Q}=\mathrm{O}$ representing the unperturbed Hamiltonian. When compared to the same calculation performed on the divacancy (not shown), this term is about two order of magnitude higher.

\section{Discussion}

Rationale for extended wave functions: The large spatial extension is due to the proximity of the energies of the electronic states, which causes the wave functions of the different defects to mix by forbidden crossing and thus to form a linear combination of the separate 'pure' defects. Therefore, by comparison with point defects for which the addition or removal of an electron radically modify one single bond, several bonds are slightly modify in a cluster, thus decreasing the displacement $\Delta \mathrm{Q}$ needed to make the transition.

Large variations of generation rate: The electronic trap the closest to the middle of the mid-gap, i.e. the one producing the higher DC and noted here $E_{t}^{m g}$ (as mid-gap) is the most detrimental one for the component. The change in atomic position between metastable configurations can occur anywhere in the defect group. Moreover, each defect is responsible for 

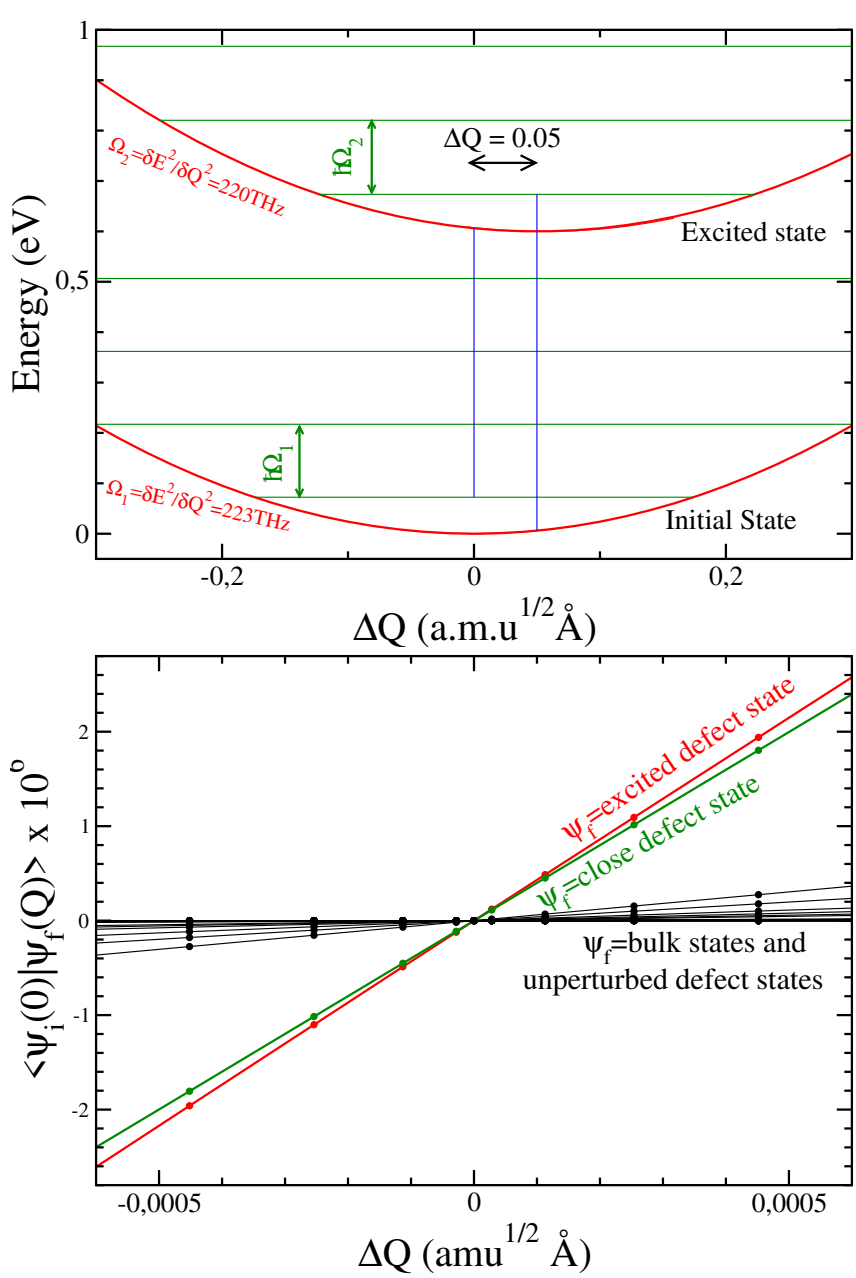

Fig. 4: Top panel: Variation of the energy of the cluster along the path $\Delta \mathbf{Q}$ for the excited and final states. Bottom panel: Variation of the scalar product $\left\langle\phi_{i}(0) \mid \phi_{f}(\mathbf{Q})\right\rangle$ along the path $\Delta \mathrm{Q}$.

its own trap level $E_{t}$. This could imply that each switch of the Fig. 1 only changes its own $E_{t}$ levels in the band gap. with a very low probability that this level corresponds to $E_{t}^{m g}$. However, with cross sections four orders of magnitude higher for clusters than for point defects, a variation of $E_{t}^{m g}$ even as small as $0.01 \mathrm{eV}$ can drastically modify the electron generation rate (exponential part of the Eq. 1), thus obtaining the difference of thousands of electrons per second between two DCs of distinct structures (observation d). The fact that a very small variation of $E_{t}^{m g}$ is sufficient for such DC changes implies that it is not necessary that the atomic structure change occurs exactly on the atoms that create this trap. In fact, the small variation in $E_{t}^{m g}$ can be induced by the change in longrange elastic distortions that create the structure change of any sufficiently close defect.

Other symmetric energy barriers: As shown in Fig. 1, many energy barriers are possible. However, only a few of them are symmetric: those with final-initial energy completely zero. All the other ones have a lifetime ratio $r_{T}$ that is too large to be observable.

Among the other possible symmetric energy barriers, any rotation of single defects must also be taken into account if they are close enough to the cluster to induce a change in $E_{t}^{m g}$. The symmetry of the rotation can be slightly modified by the nearby cluster implying that $r_{T}$ is not exactly equal to one (observation $g$ ). Moreover, many simple defects such as the tetra or penta vacancy [36], or the E center [13] can rotate on themselves without diffusing, allowing to go through six or more identical topologies in a few seconds, corresponding to observations $b$ and $g$ simultaneously.

\section{CONClusion}

We have shown that clusters of interstitial silicon atoms can produce RTS signals in agreement with observations $a$ to $d$. The present calculations tend to indicate that the presence of dopants is not necessary to generate high DC RTS, which is also consistent with observation $e$. The electric field does not play a role in our structure modification hypothesis, which is consistent with observation $f$. Finally, although observation $g$ is likely biased by experimental time scales, it can be described by any topology-preserving structure modification acting as an $E_{t}^{b g}$ modulator.

\section{ACKNOWLEDGEMENTS}

This work was granted access to the HPC resources of CALMIP-Grant P1555 and of TGCC-Irene under the allocation A0090911942 made by GENCI. This work was partially supported by IPCEI "Nano2022" program. The research leading to these results has received funding from the European Union's Horizon 2020 research and innovation program under grant agreement No. 871813 MUNDFAB. A. Jay, N. Richard, S. De Gironcoli, L. Martin-Samos and A. Hémeryck are active members of the Multiscale and Multi-Model Approach for Materials in Applied Science consortium (MAMMASMIAS consortium), and acknowledge the efforts of the consortium in fostering scientific collaboration.

\section{REFERENCES}

[1] K. Huang and A. Rhys, "Theory of light absorption and non-radiative transitions in f-centres,' Proc. Roy. Soc. A, vol. 204, pp. 406-423, 1950.

[2] W. Shockley and W. Read, "Statistics of the recombinations of holes and electrons," Phys. Rev., vol. 87, no. 5, pp. 835-842, 1952.

[3] R. N. Hall, "Electron-hole recombination in germanium," Phys. Rev., vol. 87, p. $387,1952$.

[4] C. Durnez, V. Goiffon, C. Virmontois, J. Belloir, P. Magnan, and L. Rubaldo, "In-depth analysis on radiation induced multi-level dark current random telegraph signal in silicon solid state image sensors," IEEE Trans. Nucl. Sc., vol. 64, pp. 19-26, 2017.

[5] Y. Mori, K. Takeda, and R.-i. Yamada, "Random telegraph noise of junction leakage current in submicron devices," Journal of Applied Physics, vol. 107, no. 1, p. 014509, 2010.

[6] Y. Mori, H. Yoshimoto, K. Takeda, and R.-i. Yamada, "Mechanism of random telegraph noise in junction leakage current of metal-oxidesemiconductor field-effect transistor," Journal of Applied Physics, vol. 111, no. 10, p. 104513, 2012.

[7] E. Simoen and C. Claeys, Random Telegraph Signals in Semiconductor Devices, ser. 2053-2563. IOP Publishing, 2016. [Online]. Available: http://dx.doi.org/10.1088/978-0-7503-1272-1

[8] S. Hsu, R. Whittier, and C. Mead, "Physical model for burst noise in semiconductor devices," Solid-State Electronics, vol. 13, no. 7, pp. 1055-1071, 1970 
[9] D. Pogany, J. A. Chroboczek, and G. Ghibaudo, "Random telegraph signal noise mechanisms in reverse base current of hot carrier-degraded submicron bipolar transistors: Effect of carrier trapping during stress on noise characteristics," Journal of Applied Physics, vol. 89, no. 7, pp. 4049-4058, 2001

[10] I. Hopkins and G. Hopkinson, "Further measurements of random telegraph signals in proton irradiated ccds," IEEE Transactions on Nuclear Science, vol. 42, no. 6, pp. 2074-2081, 1995.

[11] T. Nuns, G. Quadri, J.-P. David, and O. Gilard, "Annealing of protoninduced random telegraph signal in ccds," IEEE Transactions on Nuclear Science, vol. 54, no. 4, pp. 1120-1128, 2007.

[12] F. Di Capua, M. Campajola, L. Campajola, C. Nappi, E. Sarneli, L. Gasparini, and H. Xu, "Random telegraph signal in proton irradiated single-photon avalanche diodes," IEEE Trans. Nucl. Sc., vol. 65, no. 8, pp. 1654-1660, 2018.

[13] G. Herrero-Saboya, L. Martin-Samos, A. Jay, A. Hemeryck, and N. Richard, "A comprehensive theoretical picture of e centers in silicon: From optical properties to vacancy-mediated dopant diffusion," J. App. Phys., vol. 127, p. 085703, 2020.

[14] T. Umeda, K. Okonogi, K. Ohyu, S. Tsukada, K. Hamada, S. Fujieda, and Y. Mochizuki, "Single silicon vacancy-oxygen complex defect and variable retention time phenomenon in dynamic random access memories," Applied Physics Letters, vol. 88, no. 25, p. 253504, 2006.

[15] K. Ohyu, T. Umeda, K. Okonogi, S. Tsukada, M. Hidaka, S. Fujieda, and Y. Mochizuki, "Quantitative identification for the physical origin of variable retention time: A vacancy-oxygen complex defect model," in 2006 International Electron Devices Meeting, 2006, pp. 1-4.

[16] V. Goiffon, T. Bilba, T. Deladerrière, G. Beaugendre, A. Le Roch, A. Dion, C. Virmontois, J.-M. Belloir, M. Gaillardin, A. Jay, and P. Paillet, "Radiation-induced variable retention time in dynamic random access memories," IEEE Trans. Nucl. Sc., vol. 67, no. 1, pp. 234-244, 2020.

[17] M. Raine, V. Goiffon, P. Paillet, O. Duhamel, S. Girard, M. Gaillardin, C. Virmontois, J. Belloir, N. Richard, and P. Magnan, "Exploring the kinetics of formation and annealing of single particle displacement damage in microvolumes of silicon," IEEE Trans. Nucl. Sc., vol. 61, pp. 2826-2833, 2014.

[18] C. Durnez, V. Goiffon, C. Virmontois, P. Magnan, and L. Rubaldo, "Comparison of dark current random telegraphsignals in silicon and insb-basedphotodetector pixel arrays," IEEE Trans. Nucl. Sc., vol. 67, pp. 92-100, 2020.

[19] J. M. Belloir, V. Goiffon, C. Virmontois, P. Paillet, M. Raine, R. Molina, C. Durnez, , O. Gilard, and P. Magnan, "Dark current spectroscopy on alpha irradiated pinned photodiode cmos image sensors,' IEEE Trans. Nucl. Sc., vol. 63, pp. 2183-2192, 2016.

[20] S. J. Watts et al., "A new model for generation-recombination in silicon depletion regions after neutron irradiation," IEEE Trans. Nucl. Sc., vol. 43, pp. 2587-2594, 1997.

[21] J. Bourgoin and M. Lannoo, Point Defects in Semiconductors II. Springer Berlin Heidelberg, 1983.

[22] A. Le Roch, C. Virmontois, P. Paillet, J. M. Belloir, S. Rizzolo, O. Marcelot, H. Dewitte, M. Van Uffelen, L. Mont Casellas, P. Magnan, and V. Goiffon, "Phosphorus versus arsenic: Role of the photodiode doping element in cmos image sensor radiation-induced dark current and random telegraph signal," IEEE Trans. Nucl. Sc., vol. 7, pp. 12411250,2020

[23] A. Le Roch, C. Virmontois, P. Paillet, J. Belloir, S. Rizzolo, F. Pace, C. Durnez, P. Magnan, and V. Goiffon, "Radiation-induced leakage current and electric field enhancement in cmos image sensor sense node floating diffusions," IEEE Trans. Nucl. Sc., vol. 66, no. 3, pp. 616-624, 2019.

[24] K. Jóźwikowski, M. Kopytko, A. Rogalski, and A. Jóźwikowska, "Enhanced numerical analysis of current-voltage characteristics of long wavelength infrared n-on-p hgcdte photodiodes," J. App. Phys., vol. 108, p. $074519,2010$.

[25] C. Durnez, V. Goiffon, C. Virmontois, S. Rizzolo, A. Le Roch, P. Magnan, P. Paillet, C. Marcandella, and L. Rubaldo, "Total ionizing dose radiation-induced dark current random telegraph signal in pinned photodiode cmos image sensors," IEEE Trans. Nucl. Sc., vol. 65, pp. 92-100, 2018

[26] A. Jay, M. Raine, N. Richard, N. Mousseau, V. Goiffon, A. Hémeryck, and P. Magnan, "Simulation of single particle displacement damage in silicon-part ii: Generation and long-time relaxation of damage structure," IEEE Trans. Nucl. Sc., vol. 64, pp. 141-148, 2017.
[27] A. Jay, A. Hmeryck, N. Richard, L. Martin-Samos, M. Raine, A. Le Roch, N. Mousseau, V. Goiffon, P. Paillet, M. Gaillardin, and P. Magnan, "Simulation of single particle displacement damage in silicon-part iii: First principle characterization of defect properties," IEEE Trans. Nucl. Sc., vol. 65, pp. 724-731, 2018.

[28] B. J. Coomer, J. P. Goss, R. Jones, S. Oberg, and P. R. Briddon, "Interstitial aggregates and a new model for the i1/w optical centre in silicon," Physica B, vol. 273-274, pp. 505-508, 1999.

[29] N. E. B. Cowern, G. Mannino, P. A. Stolk, F. Roozeboom, H. G. A Huizing, J. G. M. van BerkumPhilips, F. Cristiano, A. Claverie, and M. Jaraiz, "Energetics of self-interstitial clusters in si," Phys. Rev. Let., vol. 82, no. 22, pp. 4460-4463, 1999.

[30] L. A. Marquès, M. Aboy, M. Ruiz, I. Santos, P. López, and P. Pelaz, "001 loops in silicon unraveled," Acta Mater., vol. 166, pp. 192-201, 2019.

[31] S. Plimton, "Fast parallel algorithms for short-range molecular dynamics," J. Chem. Phys., vol. 117, pp. 1-19, 1995. [Online]. Available: http://lammps.sandia.gov

[32] F. El-Mellouhi, N. Mousseau, and L. Lewis, "Kinetic activationrelaxation technique: An off-lattice self-learning kinetic monte carlo algorithm," Phys. Rev. B, vol. 78, p. 153202, 2008.

[33] A. Jay, C. Huet, N. Salles, M. Gunde, L. Martin-Samos, N. Richard, G. Landa, V. Goiffon, S. De Gironcoli, A. Hémeryck, and N. Mousseau, "Finding reaction pathways and transition states: r-arth and dartn as an efficient and versatile alternative to string approaches," J. Chem. Theory Comput., vol. 16, pp. 6726-6734, 2020.

[34] A. Alkauskas, Q. Yan, and C. V. de Walle, "First-principles theory of nonradiative carrier capture via multiphonon emission," Phys. Rev. B, vol. 90, p. 075202, 2014.

[35] G. H. Vineyard, "Frequency factors and isotope effects in solid state rate processes," J. Phys. Chem. Sol., vol. 3, pp. 121-127, 1957.

[36] D. V. Makhov and L. Lewis, "Stable fourfold configurations for small vacancy clusters in silicon from ab initio calculations," Phys. Rev. Let. vol. 92, no. 25, p. 255504, 2004. 